University of Wollongong

Research Online

Australian Institute for Innovative Materials -

Papers

Australian Institute for Innovative Materials

$1-1-2014$

Exchange interaction mediated ferroelectricity in multiferroic MnTiO3 with anisotropic orbital hybridization and hole delocalization

ShiWei Chen

National Synchrotron Radiation Research Center

Paoan Lin

National Tsing Hua University

Horngtay Jeng

National Tsing Hua University

$\mathrm{SW}$. Fu

National Synchrotron Radiation Research Center

Jennmin Lee

National Synchrotron Radiation Research Center

See next page for additional authors

Follow this and additional works at: https://ro.uow.edu.au/aiimpapers

Part of the Engineering Commons, and the Physical Sciences and Mathematics Commons

Research Online is the open access institutional repository for the University of Wollongong. For further information contact the UOW Library: research-pubs@uow.edu.au 


\title{
Exchange interaction mediated ferroelectricity in multiferroic MnTiO3 with anisotropic orbital hybridization and hole delocalization
}

\begin{abstract}
We present the orbital structure of $\mathrm{MnTiO} 3$ with polarization dependent $\mathrm{x}$-ray absorption and resonant $\mathrm{x}$ ray emission spectra accompanied with electronic structure calculations. The results clearly indicate a strongly anisotropic $02 \mathrm{p}-\mathrm{Mn} 3 \mathrm{~d}$ orbital hybridization whereas the $\mathrm{Mn} 3 \mathrm{~d}$ hole state shows a highly delocalized characteristic ascribed to the $3 d-4 p$ mixing. The extended $M n 4 p$ orbital could enhance the exchange interaction between $\mathrm{Mn}(3 \mathrm{~d})-\mathrm{O}(2 \mathrm{p})-\mathrm{Mn}$ (3d) leading to an asymmetric charge distribution in $\mathrm{Mn}-\mathrm{O}$ bonds. The delocalized characteristic of $\mathrm{Mn} 3 \mathrm{~d}$ holes is indispensable to the mechanism of spindependent-metal-ligand hybridization to explain magnetically induced ferroelectricity.
\end{abstract}

\section{Keywords}

anisotropic, orbital, hybridization, hole, exchange, delocalization, interaction, multiferroic, ferroelectricity, mediated, mntio3

\section{Disciplines}

Engineering | Physical Sciences and Mathematics

\section{Publication Details}

Chen, S. W., Lin, P. A., Jeng, H. T., Fu, S. W., Lee, J. M., Lee, J. F., Pao, C. W., Ishii, H., Tsuei, K. D., Hiraoka, N., Chen, D. P., Dou, S. X., Wang, X. L., Lu, K. T. \& Chen, J. M. (2014). Exchange interaction mediated ferroelectricity in multiferroic $\mathrm{MnTiO} 3$ with anisotropic orbital hybridization and hole delocalization. Applied Physics Letters, 104 (8), 082104-1-082104-5.

\section{Authors}

ShiWei Chen, Paoan Lin, Horngtay Jeng, S W. Fu, Jennmin Lee, Jyhfu Lee, Chihwen Pao, Hirofumi Ishii, Kuding Tsuei, Nozomu Hiraoka, Dapeng Chen, S X. Dou, Xiaolin Wang, Kueihtzu Lu, and Jinming Chen 


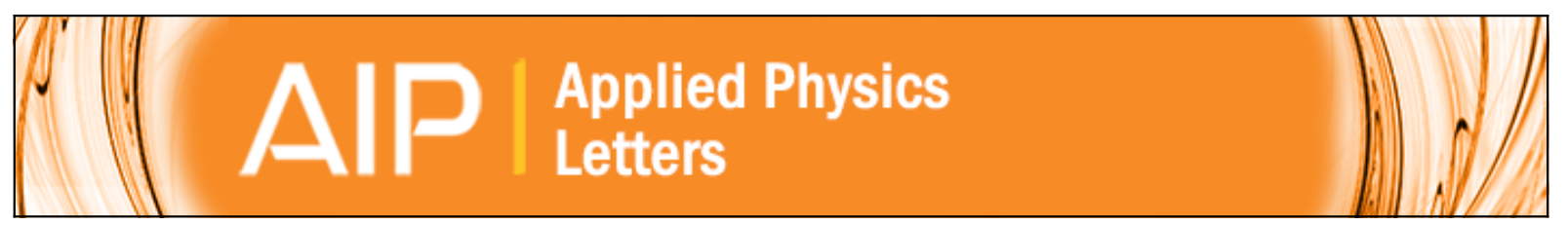

\section{Exchange interaction mediated ferroelectricity in multiferroic MnTiO3 with anisotropic orbital hybridization and hole delocalization}

S. W. Chen, P. A. Lin, H. T. Jeng, S. W. Fu, J. M. Lee, J. F. Lee, C. W. Pao, H. Ishii, K. D. Tsuei, N. Hiraoka, D.

P. Chen, S. X. Dou, X. L. Wang, K. T. Lu, and J. M. Chen

Citation: Applied Physics Letters 104, 082104 (2014); doi: 10.1063/1.4866462

View online: http://dx.doi.org/10.1063/1.4866462

View Table of Contents: http://scitation.aip.org/content/aip/journal/apl/104/8?ver=pdfcov

Published by the AIP Publishing

\section{Articles you may be interested in}

Coexistence of four resistance states and exchange bias in La0.6Sr0.4MnO3/BiFeO3/La0.6Sr0.4MnO3

multiferroic tunnel junction

Appl. Phys. Lett. 104, 043507 (2014); 10.1063/1.4863741

Magnetoelectric coupling driven by inverse magnetostriction in multiferroic BiMn3Mn4O12

J. Appl. Phys. 113, 043920 (2013); 10.1063/1.4789350

Magnetoelectric effect and critical thickness for ferroelectricity in $\mathrm{Co} / \mathrm{BaTiO} / \mathrm{Co}$ multiferroic tunnel junctions

J. Appl. Phys. 109, 114107 (2011); 10.1063/1.3587172

Static and dynamic properties of hexagonal multiferroic $\mathrm{R}$ MnO 3 compounds

J. Appl. Phys. 102, 043911 (2007); 10.1063/1.2767377

Synthesis and characterization of an $n=6$ Aurivillius phase incorporating magnetically active manganese, Bi 7 ( $\mathrm{Mn}, \mathrm{Ti}$ ) 6 O 21

Appl. Phys. Lett. 91, 033113 (2007); 10.1063/1.2756163

\section{NEW! Asylum Research MFP-3D Infinity ${ }^{\mathrm{m}}$ AFM}

Unmatched Performance, Versatility and Support

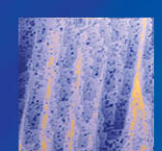

Comprehensive tools for nanomechanics
Stunning high performance
Simpler than ever to GetStarted ${ }^{\mathrm{TM}}$

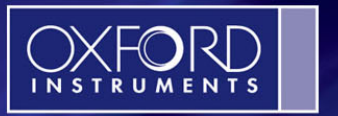

The Business of Science ${ }^{\mathrm{e}}$

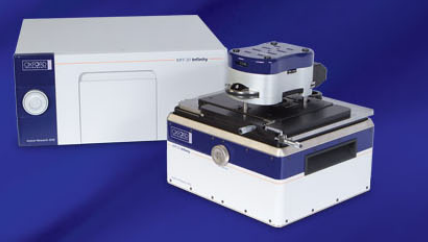




\title{
Exchange interaction mediated ferroelectricity in multiferroic $\mathrm{MnTiO}_{3}$ with anisotropic orbital hybridization and hole delocalization
}

\author{
S. W. Chen, ${ }^{1}$ P. A. Lin, ${ }^{2}$ H. T. Jeng,${ }^{2,3, a)}$ S. W. Fu, ${ }^{1}$ J. M. Lee, ${ }^{1}$ J. F. Lee, ${ }^{1}$ C. W. Pao, ${ }^{1}$ \\ H. Ishii, ${ }^{1}$ K. D. Tsuei, ${ }^{1}$ N. Hiraoka, ${ }^{1}$ D. P. Chen, ${ }^{4}$ S. X. Dou, ${ }^{4}$ X. L. Wang, ${ }^{4, a)}$ K. T. Lu, ${ }^{1}$ \\ and J. M. Chen ${ }^{1, a)}$ \\ ${ }^{1}$ National Synchrotron Radiation Research Center, Hsinchu 30076, Taiwan \\ ${ }^{2}$ Department of Physics, National Tsing Hua University, Hsinchu 30013, Taiwan \\ ${ }^{3}$ Institute of Physics, Academia Sinica, Taipei 11529, Taiwan \\ ${ }^{4}$ Institute for Superconducting and Electronic Materials, Australian Institute of Innovative, \\ Materials, University of Wollongong, North Wollongong, NSW 2500, Australia
}

(Received 13 November 2013; accepted 9 February 2014; published online 25 February 2014)

\begin{abstract}
We present the orbital structure of $\mathrm{MnTiO}_{3}$ with polarization dependent x-ray absorption and resonant $\mathrm{x}$-ray emission spectra accompanied with electronic structure calculations. The results clearly indicate a strongly anisotropic $\mathrm{O} 2 p$-Mn $3 d$ orbital hybridization whereas the $\mathrm{Mn} 3 d$ hole state shows a highly delocalized characteristic ascribed to the $3 d-4 p$ mixing. The extended $\mathrm{Mn} 4 p$ orbital could enhance the exchange interaction between $\mathrm{Mn}(3 d)-\mathrm{O}(2 p)$-Mn $(3 d)$ leading to an asymmetric charge distribution in Mn-O bonds. The delocalized characteristic of $\mathrm{Mn} 3 d$ holes is indispensable to the mechanism of spin-dependent-metal-ligand hybridization to explain magnetically induced ferroelectricity. (C) 2014 AIP Publishing LLC.

[http://dx.doi.org/10.1063/1.4866462]
\end{abstract}

Magnetoelectric (ME) effects, through which magnetism and ferroelectricity are intimately coupled, remain of great interest, in part due to the discovery of new multiferroic materials as well as gigantic ferroelectricity induced by a frustrated magnetic ordering. For a substance such as E-type antiferromagnetic (AFM) $\mathrm{HoMnO}_{3}$ the competing magnetic-exchange interactions, of similar magnitude, produce unconventional spin states that break inversion symmetry. The doubleexchange interaction, asymmetric electron hopping of orbitally polarized $e_{g}$ states that produces a polar charge distribution, thus drives the magnetically induced ferroelectric dipole, arising even without atomic displacements. ${ }^{1}$

The microscopic mechanism of magnetically induced ferroelectricity in AFM-E manganites can be realized by the spin-dependent metal $(d)$-ligand $(p)$ hybridization, based on the strong spin-orbit coupling. ${ }^{1,2}$ The altering angular dependence of the $d$-orbital wave functions and hence the electron density via the $p$ - $d$ hybridization might generate an electric dipole moment, similar to the procedure in the charge transfer model. ${ }^{3}$ In that situation, the degree of delocalization of transition-metal $d$ holes is of crucial importance. Only when the transition metal $d$ holes exhibit a delocalized characteristic, the mechanism of spin-dependent metalligand hybridization can be tenable. However, little investigation has been devoted to study comprehensively its influence on the exchange interaction as well as the induced ferroelectric dipole. The $d$ orbital degree of freedom, localization, or delocalization has not been verified yet, probably due to the difficulty in the measurements. As established, resonant $\mathrm{x}$-ray emission spectroscopy (RXES) is a powerful technique to provide detailed information about the degree

\footnotetext{
${ }^{\text {a) }}$ Authors to whom correspondence should be addressed. Electronic addresses: jeng@phys.nthu.edu.tw; xiaolin@uow.edu.au; and jmchen@nsrrc.org.tw.
}

of delocalization of the unoccupied states or hole carriers in materials. ${ }^{4,5}$ The technique is thus utilized in this study.

Herein, we focus on the material of $\mathrm{MnTiO}_{3}$ because it is another compound that shows magnetically controlled electric polarization with the linear magnetoelectric effect. ${ }^{6,7}$ Similarly, this effect occurs as inversion symmetry breaking. The electric polarization $(\mathrm{P})$ is zero unless and external magnetic field $(\mathrm{H})$ is applied. In the presence of a magnetic field, $\mathrm{P}$ increases proportionally to $\mathrm{H}$, and correspondingly, magnetization $\mathrm{M}$ increases linearly with applied electric field $\mathrm{E}$. Both effects are deducible from the macroscopic free energy term $\mathrm{F}(\mathrm{L}, \mathrm{E}, \mathrm{H})$, in which $\mathrm{L}$ is the antiferromagnetic order parameter. ${ }^{8}$ However, the microscopic mechanism of giant ferroelectricity in $\mathrm{MnTiO}_{3}$ is even less understood. We thus intend to characterize the $\mathrm{Mn} 3 d$ orbital of $\mathrm{MnTiO}_{3}$ with experiments and calculations, especially for $\mathrm{Mn} 3 d-\mathrm{O} 2 p$ and Mn $3 d$-Mn $4 p$ orbital hybridizations. With a clear framework of orbital construction and identification of orbital degree of freedom, the microscopic mechanism of ferroelectricity in $\mathrm{MnTiO}_{3}$ is discussed clearly in this study.

In this work, $\mathrm{MnTiO}_{3}$ single crystals were synthesized with a four-mirror floating-zone method. The rhombohedral structure, R-3, of single-crystalline $\mathrm{MnTiO}_{3}$ was characterized with four-axis $\mathrm{x}$-ray diffraction according to which a periodic diffraction pattern was identified. Polarization dependent $\mathrm{O}$ $K$-edge and Ti $K$-edge x-ray absorption near edge structure (XANES) spectra were probed at National Synchrotron Radiation Research Center (NSRRC) in Taiwan. O $K$-edge spectra were recorded by collecting surface-electron (total electron yield, TEY) and fluorescence (total fluorescence yield, TFY) signals simultaneously to confirm the consistency between the surface and the bulk. Figure 1(a) shows $O K$-edge XANES spectra of $\mathrm{MnTiO}_{3}$ with polarization $E$ perpendicular $(E / / \mathrm{c})$ and nearly parallel (E//ab plane) to the ab-plane. Above the absorption threshold, two pronounced features, $\mathrm{A}$ and $\mathrm{B}$, 

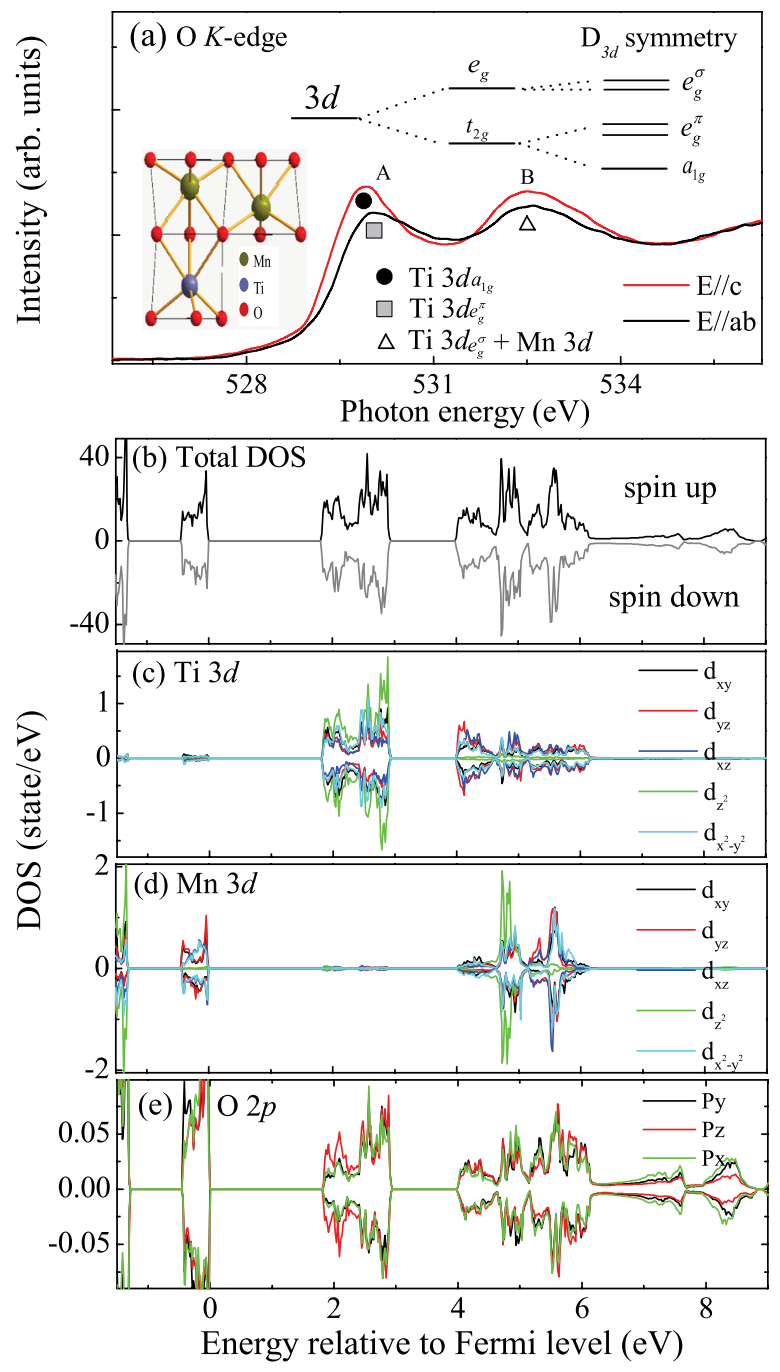

FIG. 1. (a) Polarization dependent $\mathrm{O} K$-edge XANES spectra of $\mathrm{MnTiO}_{3}$; (b) the calculated total density of state (DOS); the decomposed DOS of (c) $\mathrm{Ti}$, (d) Mn, and (e) O. DOS are consisted of spin up and spin components as indicated.

are displayed. Feature $\mathrm{A}$ is situated at a smaller energy for $E / / \mathrm{c}$ with an intensity significantly greater than for the $E / /$ ab plane. Feature $\mathrm{B}$ also exhibits a greater intensity for the $E / / \mathrm{c}$ spectrum than for the $E / /$ ab spectrum. These large spectral variations indicate that the hybridization between $\mathrm{O} 2 p$ and Ti $3 d$, or $\mathrm{Mn}$ $3 d$, orbitals is highly anisotropic. To identify precisely the corresponding orbitals in the polarization dependent $\mathrm{O} K$-edge XANES spectra of $\mathrm{MnTiO}_{3}$, we performed calculations of the electronic structure with the accurate full-potential projected-augmented-wave method as implemented in the VASP package within the generalized-gradient approximation (GGA) as well as the GGA plus Hubbard U (GGA+U) scheme. ${ }^{9}$ The calculations were performed over a $13 \times 13 \times 5$ Monkhorst-Pack $k$-point mesh in the irreducible Brillouin zone using 72000 plane waves with energy truncated at $500 \mathrm{eV}$. On-site Coulomb energy $U=4 \mathrm{eV}$ and exchange parameter $J=0.6 \mathrm{eV}$ were used for Mn ions to explore the correlation effects in $3 d$ orbitals whereas we used $U=0, J=0$ for Ti ions because of the nearly empty valence electron shell. Figs. 1(b)-1(e) present the calculated density of states of $\mathrm{MnTiO}_{3}$ focusing on the conduction band region. These results clearly expose the $\mathrm{Ti} 3 d$ orbital, divided into the low-lying $t_{2 g}$ and high-lying $e_{g}$ states under a crystal field, contributes mainly to the electronic structure about $1.8-2.9 \mathrm{eV}$ (feature A) and 4-6.1 eV (feature B), respectively, whereas the contribution of the Mn $3 d$ state is mainly at $4-6.1 \mathrm{eV}$ (feature B) relative to the Fermi level. In $\mathrm{D}_{3 \mathrm{~d}}$ symmetry, a trigonal distortion of the $\mathrm{MO}_{6}(\mathrm{M}=\mathrm{Ti}, \mathrm{Mn})$ octahedra further splits the $t_{2 g}$ state into non-degenerate $a_{1 g}$ and doubly degenerate $e_{g}^{\pi}$ sub-states whereas the $e_{g}$ state remains doubly degenerate, now called $e_{g}^{\sigma}$. As indicated in the calculated density of states shown in Figs. 1(c) and 1(d), the splitting in the Ti $3 d$ state is much greater than that in the Mn $3 d$ state, because the local distortion in $\mathrm{TiO}_{6}$ octahedral symmetry is more severe than that in $\mathrm{MnO}_{6}$ octahedral symmetry. According to the split levels shown in the upper part of Fig. 1(a), the features in the $\mathrm{O} K$-edge spectra are satisfactorily assigned. Intense feature $\mathrm{A}$, in the polarization-dependent $\mathrm{O}$ $K$-edge XANES spectra of $\mathrm{MnTiO}_{3}$, situated at an energy for $E / / \mathrm{c}$ lower than for $E / / \mathrm{ab}$, reflects mainly a strong hybridization between $\mathrm{O} 2 p$ and Ti $3 d\left(a_{1 g}\right)$ orbitals lying near the $\mathrm{c}$ axis, and O $2 p$-Ti $3 d\left(e_{g}^{\pi}\right)$ hybridized orbital lying along the ab plane. The result is confirmed by the projected density of states of $\mathrm{O}$ $2 p$ and Ti $3 d$ orbitals as shown in Figs. 1(c) and 1(e), respectively. As noted, the $\mathrm{O} 2 p_{z}$ state, near the c axis, is significantly situated at a lower energy, coinciding with a $\mathrm{Ti} 3 d_{Z}^{2}\left(a_{1 g}\right)$ state. That condition clearly indicates a strong $\mathrm{O} 2 p_{z}$-Ti $3 d\left(a_{l g}\right)$ hybridization near the $\mathrm{c}$ axis. Feature $\mathrm{B}$ in the polarization dependent $\mathrm{O} K$-edge spectra is constructed from a combination of $\mathrm{O} 2 p$-Ti $3 d\left(e_{g}^{\sigma}\right)$ and $\mathrm{O} 2 p-\mathrm{Mn} 3 d$ hybridized states, in which the $\mathrm{O} 2 p-\mathrm{Mn} 3 d$ hybridized state with an essentially devotion is indicated by the decomposed density of states shown in Fig. 1(d). The intensity of feature B significantly greater for $E / / \mathrm{c}$ than for $E / / \mathrm{ab}$ thus reflects the stronger hybridization between $\mathrm{O} 2 p$ and $\mathrm{Mn} 3 d$ orbitals lying near the $\mathrm{c}$ axis.

Figure 2(a) shows the polarization dependent Ti $K$-edge XANES spectra of $\mathrm{MnTiO}_{3}$ recorded by collecting fluorescence emitted from the sample with a Lyttle detector. As noted, a pronounced difference in the pre-edge region and the near-edge region was observed with polarizations $E$ perpendicular or parallel to the $a b$ plane. The absorption features in the pre-edge region are ascribed to the quadrupole transition from $1 s$ to $3 d-4 p$ mixing states, upon the non-symmetric molecular structure or an additional contribution of a $4 p$ dipole characteristically mediated by $\mathrm{O}$ atoms. ${ }^{10,11}$ In Fig. 2(a), we observe a prominent feature in the pre-edge region of $\mathrm{Ti} K$-edge XANES spectra of $\mathrm{MnTiO}_{3}$, especially for polarization $E / / a b$, thus reflecting the hybridization of $\mathrm{Ti} 3 d$ and $4 p$ orbitals occurring predominantly in the $a b$ plane.

We investigated also the orientation of $\mathrm{Mn}$ orbital in $\mathrm{MnTiO}_{3}$ with polarization dependent $\mathrm{Mn} K$-edge XANES spectra. To expose evidently the feature in the pre-edge region of Mn $K$-edge spectra, spectra were recorded in the partial fluorescence yield (PFY) mode at the undulator beamline BL12U1 of Spring-8 in Japan. They were measured on tuning the spectrometer energy to the maximum of the Mn $K \beta_{1,3}$ emission line while scanning the incident photon energy across the Mn $K$-edge. The detector was oriented perpendicular to the incident beam polarization to suppress the elastic scattering peak and to improve the ratio of signal to noise. Absorption spectrum recorded by the partial fluorescence yield thus exhibits much higher resolution than that 

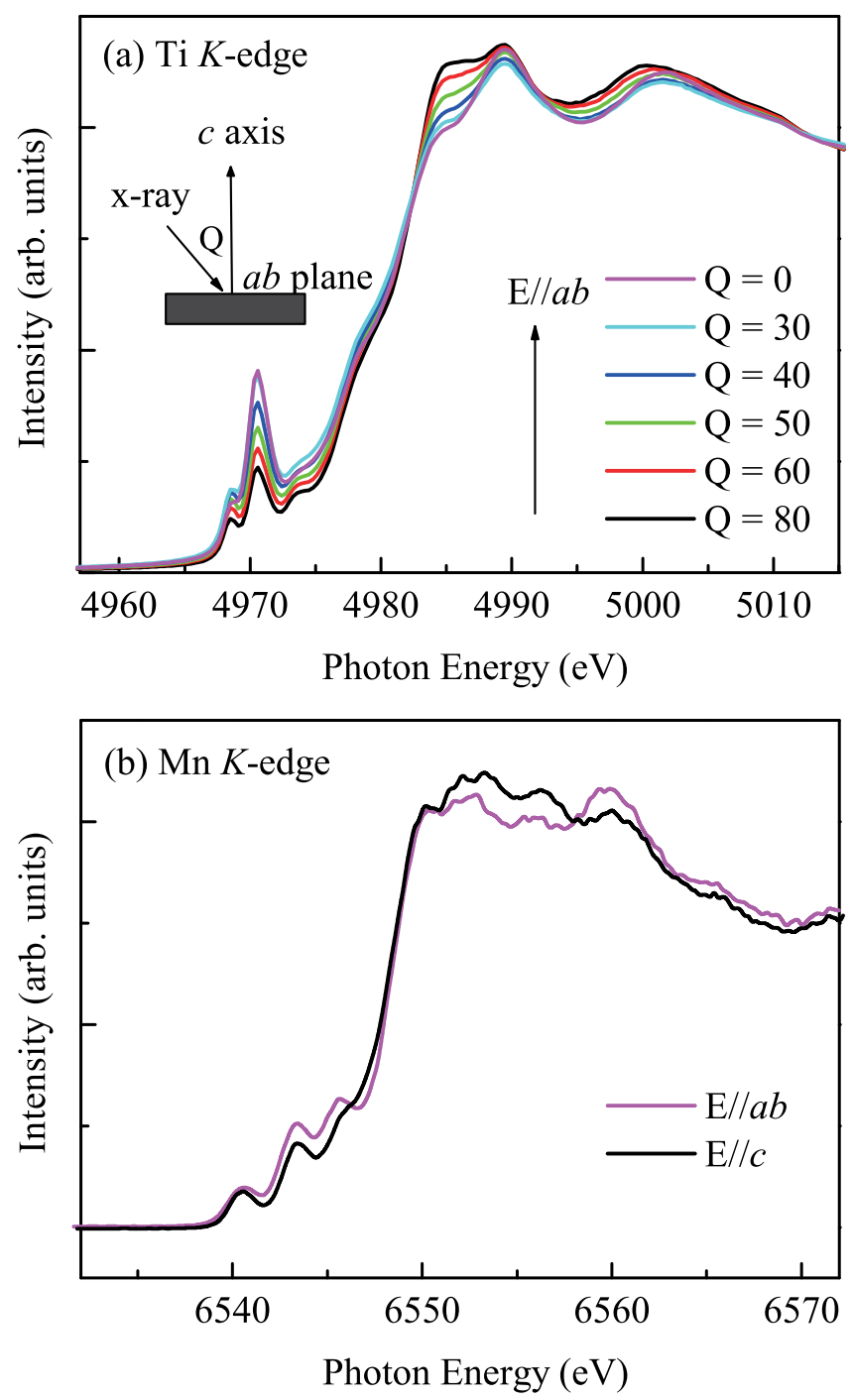

FIG. 2. (a) Polarization dependent Ti $K$-edge XANES spectra and (b) Mn $K$-edge PFY-XANES spectra of $\mathrm{MnTiO}_{3}$.

recorded as the conventional total fluorescence yield. ${ }^{12}$ Fig. 2(b) shows the polarization dependent $\mathrm{Mn} K$-edge PFY-XANES spectra of $\mathrm{MnTiO}_{3}$. Apparent features in the pre-edge region were observed despite the polarization $E$ perpendicular or parallel to the $a b$ plane, reflecting the existence of $\mathrm{Mn} 3 d / 4 p$ hybridization. One can also observe that the feature intensity is greater for polarization $E / / a b$ than for $E / / c$. That condition indicates the slightly predominant hybridization of $\mathrm{Mn} 3 d-4 p$ orbitals in the $a b$ plane.

To further explore Mn $3 d$ orbital degree of freedom in $\mathrm{MnTiO}_{3}$, RXES spectra were also recorded at the undulator beamline BL12U1 of Spring-8 in Japan. RXES spectra of $\mathrm{MnTiO}_{3}$ were recorded with various incident energies across the Mn $K$-edge. The spectra consist of characteristics in two parts named Raman (for which the transfer energy is constant) and normal fluorescence (for which the transfer energy varied with excitation energy); the former existed at a photon energy of the incident beam far below the threshold energy of $\mathrm{Mn} K$-edge excitation whereas the latter is shown purely in the photon energy far above the threshold energy. When the incident photon energy is tuned and passed through the pre-edge, rising-edge, and white-line regions of $K$-edge, it is possible to distinguish the emission due to $1 s-3 d$ (quadruple
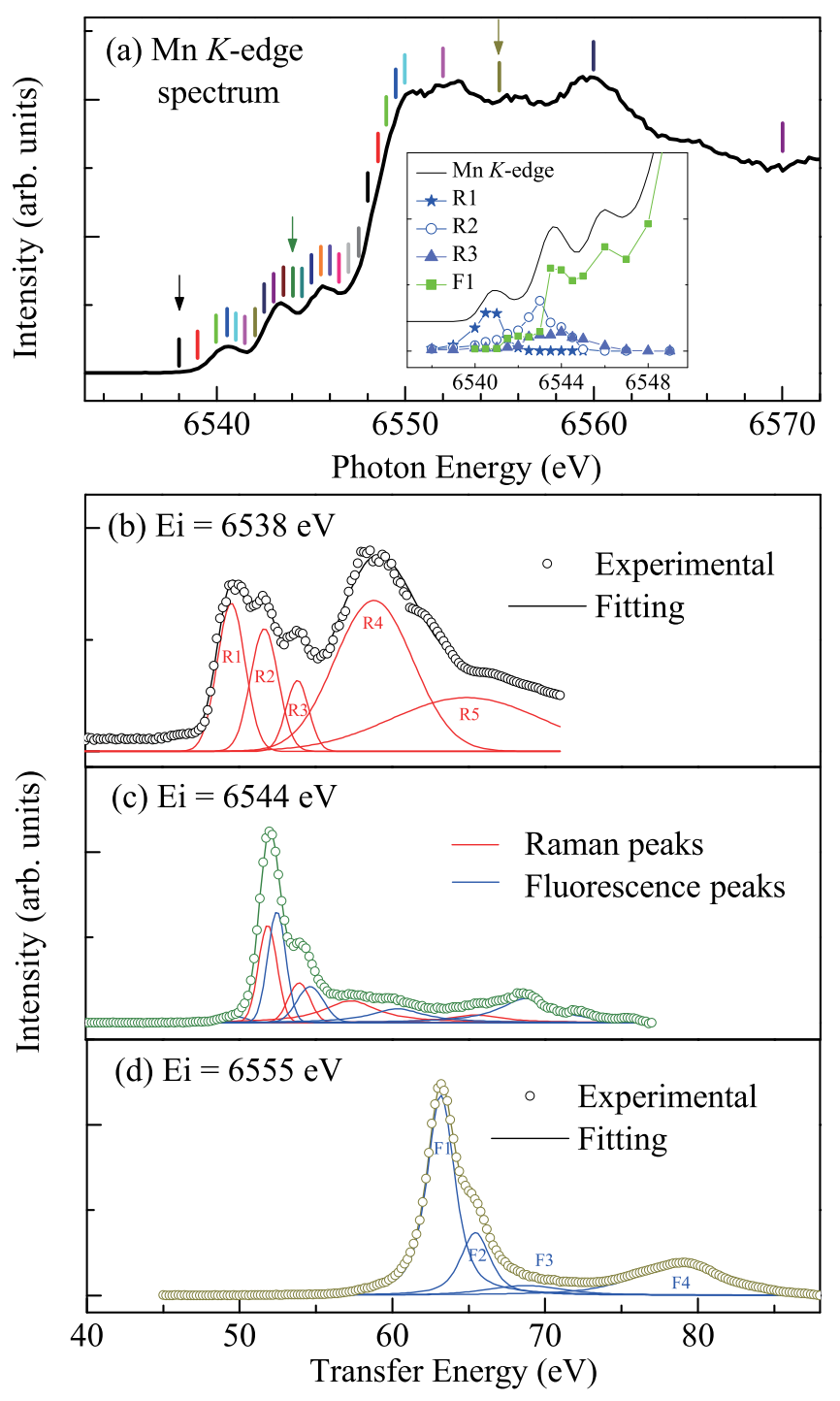

FIG. 3. (a) Mn $K$-edge PFY-XANES spectra of $\mathrm{MnTiO}_{3}$; inset shows the integration of the intensity of Raman and normal fluorescence characteristics into the pre-edge region. (b)-(d) $\mathrm{Mn} 1 s 3 p$-RXES spectra of $\mathrm{MnTiO}_{3}$ excited by different photon energy.

forbidden), $1 s-3 d / 4 p$ mixing, and $1 s-4 p$ (dipole allowed) transitions, respectively. RXES thus can provide information on the difference of electric quadrupole and dipole transitions. ${ }^{13}$ Figure 3 displays the Mn $K$-edge PFY-XANES and the RXES spectra of $\mathrm{MnTiO}_{3}$ with the incident beams at energies 6533, 6544, and $6555 \mathrm{eV}$, respectively. The pure Raman characteristics are expressed clearly in Fig. 3(b) whereas the purely normal fluorescence characteristics are shown in Fig. 3(d). For the incident energies near the pre-edge and absorption-edge of the Mn $K$-edge, the spectral characteristics of RXES spectra arise from the contributions of both Raman and normal fluorescence parts, as shown in Fig. 3(c). The inset in Fig. 3(a) further integrates the intensity of the Raman characteristic and the normal fluorescence characteristic into the pre-edge region of Mn $K$-edge XANES spectra. As indicated, the Raman regime is limited to a few eV $(\sim 5 \mathrm{eV})$ in the pre-edge region, before the fluorescence regime begins. Normal fluorescence characteristics survive at even a lower excitation energy identifying clearly the delocalization of $\mathrm{Mn} 3 d$ hole states in $\mathrm{MnTiO}_{3}$. 
Otherwise, if the normal fluorescence regime begins at a large energy, the localized character of the metal $3 d$ orbital is denoted. ${ }^{5}$ Mn $3 d$ hole states with a highly delocalized characteristic are attributed to the mixing of $3 d / 4 p$ states because the Mn $4 p$ state, being very broad, might extend even over several atoms. ${ }^{4,14}$

As demonstrated in references, $\mathrm{MnTiO}_{3}$ exhibits a linear magnetoelectric effect with the easy axis along the $c$ axis below $T_{\mathrm{N}} \sim 64 \mathrm{~K} .{ }^{6}$ Ferroelectric polarization increases linearly with applied magnetic field along the $c$-axis followed by an abrupt suppression above $H \mathrm{c}$. The sharply suppressed ferroelectricity was attributed to spin flopping. ${ }^{7}$ There are several microscopic mechanisms for ferroelectricity, including the spin-current, ${ }^{2,15}$ Dzyaloshinskii-Moriya (DM) interaction, ${ }^{16}$ and exchange striction mechanisms. ${ }^{2}$ But $\mathrm{MnTiO}_{3}$ exhibits an antiferromagnetic ordering, ${ }^{7,17}$ with a small $D$ value (mutual cancellation) and a weak spin-lattice coupling. ${ }^{6}$ Three mechanisms mentioned above are thus not useful in $\mathrm{MnTiO}_{3}$.

Magnetically induced ferroelectricity in $\mathrm{MnTiO}_{3}$ can currently be explained with spin-dependent metal-ligand hybridization, ${ }^{2,18,19}$ i.e., the $p$ - $d$ hybridization between the transition metal $(\mathrm{M})$ and the ligand $(\mathrm{O})$. Because of the hybridization relevant to the spin-orbit interaction, ionic charge $\rho$ of the ligand can vary depending on the angle $\eta$ between the spin of the transition metal and vector $e$ connecting the transition metal and the ligand, i.e., $\Delta \rho \propto(S \cdot e)^{2}$. A local electric polarization $\Delta P \propto(S \cdot e)^{2} e$ thus exists between the transition metal and the ligand. ${ }^{19}$ In this work, we have found evidence for a strongly asymmetric O $2 p$-Mn $3 d$ (see Fig. 1) and Mn $3 d-4 p$ (see Fig. 3) hybridization in $\mathrm{MnTiO}_{3}$. O $2 p$-Mn $3 d$ hybridization predominantly lies near the $c$ axis in the slightly distorted $\mathrm{MnO}_{6}$ octahedra in the slightly distorted $\mathrm{MnO}_{6}$ octahedra with a small difference in long and short Mn-O bond lengths. Mn $3 d-4 p$ mixing on the $a b$ plane constructs the orbital connection between Mn ions, via O 2p; Mn $3 d$ holes thus show a delocalized characteristic. Exchange coupling in the connection of $\mathrm{Mn}(3 d)-\mathrm{O}(2 p)-\mathrm{Mn}(3 d)$, on the $a b$ plane, can thus occur especially upon the delocalized Mn $3 d$ holes. Herein, the extended Mn $4 p$ orbital, hybridized with the empty $3 d$ orbitals, may partly account for the exchange interaction between spin electrons, as that in references the transition-metal $4 p$ orbitals mediated a superexchange path. ${ }^{14,20}$ With applying magnetic field, spin, anti-parallel to the magnetic field, attempts to alter its direction, causing a spin fluctuation. Electron hopping of orbitally polarized Mn $3 d$ states then proceeds mediated by oxygen ions. ${ }^{1}$ Under strong spin-orbital coupling, an asymmetric charge distribution between $\mathrm{Mn}$ and $\mathrm{O}$ ions could thus be obtained, causing electronic polarization. The complete process is illustrated in Figure 4. The state with two holes on the oxygen ion and extra electrons on manganese ions gives a nonzero contribution to the electric dipole in the direction orthogonal to the Mn plane.

In conclusions, polarization dependent XANES and RXES spectra, accompanied with calculations of electronic structure, expose strongly anisotropic O $2 p-\mathrm{Mn} 3 d$ orbital hybridization whereas the $\mathrm{Mn} 3 d$ hole state shows a highly delocalized characteristic ascribed to the mixing of $3 d / 4 p$ orbitals. The delocalized Mn $3 d$ hole is of crucial importance

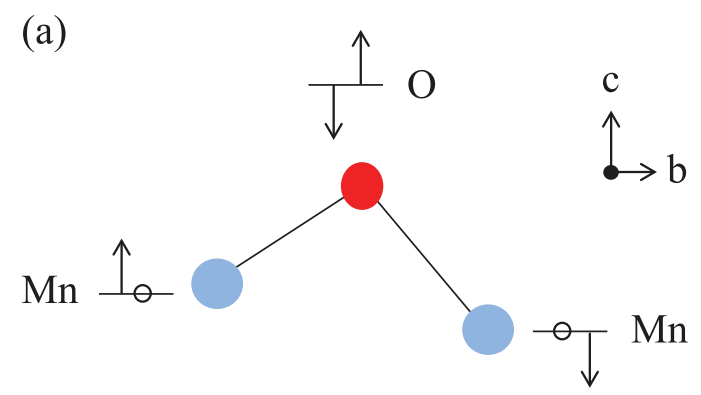

- $3 \mathrm{~d}$ delocalized hole

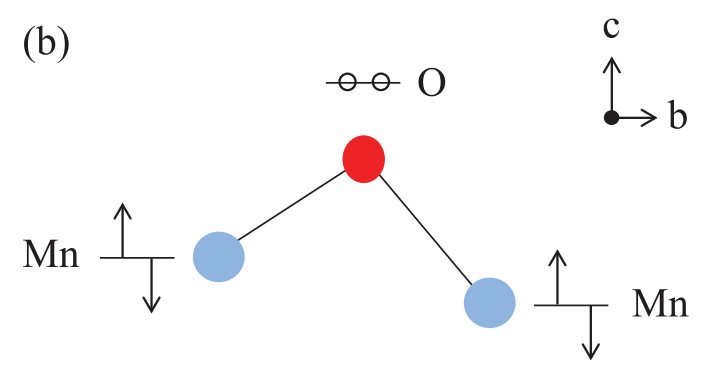

FIG. 4. Orbital connection of Mn-O-Mn ions in $\mathrm{MnTiO}_{3}$. (a) The initial state with a $3 d$ delocalized hole and a filled oxygen orbital. (b) The state with asymmetric electron distribution via spin dependent $\mathrm{Mn}$ metal-O ligand hybridization, under strong spin-orbital coupling.

to the mechanism of spin-dependent metal-ligand $(p-d)$ hybridization. Accordingly, the exchange interaction in the connection of $\mathrm{Mn}(3 d)-\mathrm{O}(2 p)-\mathrm{Mn}(3 d)$ could occur and asymmetric charge distribution in $\mathrm{Mn}-\mathrm{O}$ bonds can be obtained, under a strong spin-orbital coupling. This study evidence the delocalization of $\mathrm{Mn} 3 d$ holes in $\mathrm{MnTiO}_{3}$ that should be indispensable to the magnetically induced ferroelectricity. The results will be also of great importance to realize magnetoelectronic coupling in other multiferroic materials.

National Synchrotron Radiation Research Center and National Science Council of Republic of China (Grant No. NSC 99-2113-M-213-006-MY3) provided support of this research, and Australian Research Council supported in part through a discovery project DP0987190 and scientific visit grant from Australian Academy of Science.

${ }^{1}$ K. Yamauchi, F. Freeimuth, S. Blugel, and S. Picozzi, Phys. Rev. B 78, 014403 (2008).

${ }^{2}$ T. Arima, J. Phys. Soc. Jpn. 80, 052001 (2011).

${ }^{3}$ H. Ikeno, F. M. F. de Groot, E. Stavitski, and I. Tanaka, J. Phys.: Condens. Matter 21, 104208 (2009).

${ }^{4}$ J. M. Chen, C. K. Chen, T. L. Chou, I. Jarrige, H. Ishii, K. T. Lu, Y. Q. Cai, K. S. Liang, J. M. Lee, S. W. Huang, T. J. Yang, C. C. Shen, R. S. Liu, J. Y. Lin, H. T. Jeng, and C.-C. Kao, Appl. Phys. Lett. 91, 054108 (2007).

${ }^{5}$ J. P. Rueff, L. Journal, P. E. Petit, and F. Farges, Phys. Rev. B 69, 235107 (2004).

${ }^{6}$ N. Mufti, G. R. Blake, M. Mostovoy, S. Riyadi, A. A. Nugroho, and T. M. Palstra, Phys. Rev. B 83, 104416 (2011).

${ }^{7}$ H. Toyosaki, M. Kawasaki, and Y. Tokura, Appl. Phys. Lett. 93, 072507 (2008).

${ }^{8}$ C. Ederer and C. J. Fennie, J. Phys.: Condens. Matter 20, 434219 (2008).

${ }^{9}$ G. Kresse and J. Joubert, Phys. Rev. B 59, 1758 (1999). 
${ }^{10}$ S. W. Chen, J. M. Lee, S. Chiang, S. C. Haw, Y. C. Liang, K. T. Lu, C. W. Pao, J. F. Lee, M. T. Tang, and J. M. Chen, J. Phys. Soc. Jpn. 80, 114706 (2011).

${ }^{11}$ J. A. Sigrist, M. W. Gaultois, and A. P. Grosvenor, J. Phys. Chem. A 115, 1908 (2011).

${ }^{12}$ H. Wadati, A. J. Achkar, D. G. Hawthorn, T. Z. Regier, M. P. Singh, K. D. Truong, P. Fournier, G. Chen, T. Mizokawa, and G. A. Sawatzky, Appl. Phys. Lett. 100, 193906 (2012).

${ }^{13}$ H. Shoji, M. Taguchi, E. Hirai, T. Iwazumi, A. Kotani, S. Nanao, and Y. Isozumi, J. Phys. Soc. Jpn. 72, 1560 (2003).

${ }^{14}$ F. Bridges, C. H. Booth, M. Anderson, G. H. Kwei, J. J. Neumeier, J. Snyder, J. Mitchell, J. S. Gardner, and E. Brosha, Phys. Rev. B 63, 214405 (2001).
${ }^{15}$ H. Katsura, N. Nagaosa, and A. V. Balatsky, Phys. Rev. Lett. 95, 057205 (2005).

${ }^{16}$ X. Z. Lu, M.-H. Whangbo, S. Dong, X. G. Gong, and H. J. Xiang, Phys. Rev. Lett. 108, 187204 (2012).

${ }^{17}$ Y. Yamaguchi, T. Nakano, Y. Nozue, and T. Kimura, Phys. Rev. Lett. 108, 057203 (2012).

${ }^{18}$ A. S. Moskvin and S.-L. Drechsler, Phys. Rev. B Phys. Rev. B 78, 024102 (2008).

${ }^{19}$ H. Murakawa, Y. Onose, S. Miyahara, N. Furukawa, and Y. Tokura, Phys. Rev. Lett. 105, 137202 (2010).

${ }^{20}$ A. Bencini, I. Ciofini, and E. Giannasi, Inorg. Chem. 37, 3719 (1998). 